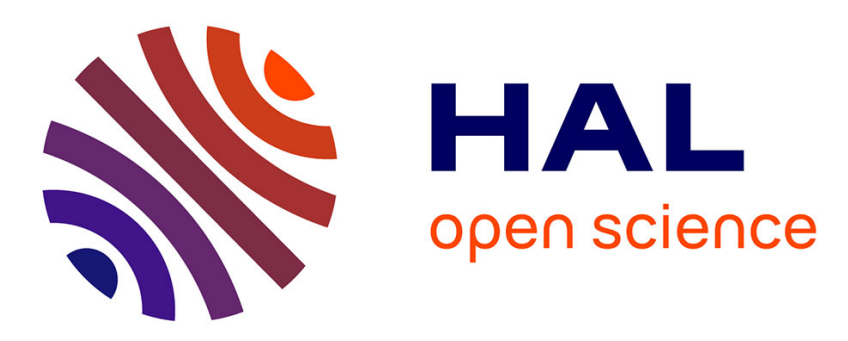

\title{
Identification of a conserved F-box protein 6 interactor essential for endocytosis cytokinesis in fission yeast
} Isabelle Jourdain, Nathalie Spielewoy, James Thompson, Susheela Dhut, John R Yates Iii, Takashi Toda

\section{- To cite this version:}

Isabelle Jourdain, Nathalie Spielewoy, James Thompson, Susheela Dhut, John R Yates Iii, et al.. Identification of a conserved F-box protein 6 interactor essential for endocytosis cytokinesis in fission yeast. Biochemical Journal, 2009, 420 (2), pp.169-177. 10.1042/BJ20081659 . hal-00479085

\section{HAL Id: hal-00479085 \\ https://hal.science/hal-00479085}

Submitted on 30 Apr 2010

HAL is a multi-disciplinary open access archive for the deposit and dissemination of scientific research documents, whether they are published or not. The documents may come from teaching and research institutions in France or abroad, or from public or private research centers.
L'archive ouverte pluridisciplinaire HAL, est destinée au dépôt et à la diffusion de documents scientifiques de niveau recherche, publiés ou non, émanant des établissements d'enseignement et de recherche français ou étrangers, des laboratoires publics ou privés. 


\title{
IDENTIFICATION OF A CONSERVED F-BOX PROTEIN 6 INTERACTOR ESSENTIAL FOR ENDOCYTOSIS AND CYTOKINESIS IN FISSION YEAST
}

\author{
Isabelle Jourdain ${ }^{1,2}$, Nathalie Spielewoy ${ }^{1,2}$, James Thompson ${ }^{3}$, Susheela Dhut ${ }^{1}$, \\ John R. Yates $\mathrm{III}^{3}$, and Takashi Toda ${ }^{1}$
}

From the ${ }^{1}$ Laboratory of Cell Regulation, Cancer Research UK, London Research Institute, 44 Lincoln's Inn Fields, London WC2A 3PX, UK, and ${ }^{3}$ the Department of Cell Biology, The Scripps Research Institute, La Jolla, California 92037

Running head: A novel conserved F-box protein interactor essential for cytokinesis

${ }^{1}$ These authors contribute equally to this work.

Address correspondence to: Takashi Toda, Laboratory of Cell Regulation, Cancer Research UK, London Research Institute, Lincoln's Inn Fields Laboratories, 44 Lincoln's Inn Fields, London WC2A 3PX, UK

E-mail: takashi.toda@cancer.org.uk

\section{Abbreviations:}

HEAT: Huntington, Elongation factor 3, the regulatory A subunit or protein phosphatase $2 \mathrm{fA}$ and Tor 1

MudPIT, Multidimensional Protein Identification Technology

SCF, Skp1-Cde53/Cullin 1-F-box protein 


\section{SYNOPSIS}

The F-box domain is a degenerated motif consisting of $\sim 40$ amino acid residues that specifically bind Skp1, a core component of the SCF (Skp1-Cdc53/Cullin 1-F-box protein) ubiquitin ligase. Recent work, mainly performed in budding yeast, indicates that certain F-box proteins form non-SCF complexes together with Skp1 in the absence of cullins and play various roles in cell cycle and signalling pathways. However it is not established whether these non-SCF complexes are unique to budding yeast or common in other eukaryotes. Here using TAP purification coupled to MudPIT analysis (Multidimensional Protein Identification Technology), we have identified a novel conserved protein, Sip1 in fission yeast, as an interacting partner of an essential F-box protein Pof6. Sip1 is a large HEAT-repeats containing protein (217 $\mathrm{kDa}$ ) and forms a complex with Pof6 and Skp1. This complex does not contain cullins, indicating that it is a novel non-SCF complex. Like Pof6 and Skp1, Sip1 is essential for cell viability and temperature sensitive sipl mutants display cell division arrest as binucleate cells with septa. Sip1 localises to the nucleus and dynamic cytoplasmic dots, which turn out to be endocytic vesicles. Consistent with this, sip1 mutants are defective in endocytosis. Furthermore towards the end of cytokinesis, constriction of the actomyosin ring and dissociation of type II myosin and septum materials are substantially delayed in the absence of functional Sip1. These results indicate that the conserved Sip1 protein comprises a novel non-SCF F-box complex that plays an essential role in endocytosis, cytokinesis and cell division.

Keywords: cytokinesis, endocytosis, F-box, fission yeast, HEAT repeats, MudPIT 


\section{INTRODUCTION}

Cytokinesis is a critical step in the cell life, as it couples the end of mitosis to the separation of the mother cell into two daughter cells. The main goal of cytokinesis is common to all organisms, and even if divergences exist, the fundamental sequences of events are universal [1]. By the end of anaphase, the fission yeasts, like animals, positions an actomyosin ring (CAR, Contractile Actomyosin Ring) in the medial cortex region and constricts it to bring about partitioning of the cytoplasm [2-5]. Concomitantly, the septum is deposited on the other side of the ring to separate the incipient daughter cells. Later a secondary septum is formed and the primary membrane is digested to allow the physical separation into two daughter cells. For these equatorial components including CAR, lipid rafts and septum to undergo assembly and dissolution in concert upon cytokinesis, membrane trafficking systems such as endocytosis, exocytosis and protein transport play vital roles. Accordingly not only actomyosin and glucans are essential in cytokinesis, but also a much larger set of conserved proteins are required for this process [2-5]. For example, the anillin-like protein Mid1, in S. pombe and other organisms, defines the position of the CAR, and the protein phosphatase Clp1/Flp1, related to budding yeast Cdc14, coordinates cytokinesis with cell cycle progression. Being simple entities, fission yeast provides the significant advantage of combining genetic and biochemistry techniques; such features make them a powerful model by which to study cytokinesis. Consistently, a precise timing of the process has been reported [6] and a plethora of mutants with defects in cytokinesis and septation have been isolated [3].

The F-box proteins were discovered for their essential role in cell cycle progression [7]. The F-box proteins are defined by the presence of a degenerated stretch of 40 amino acids, the F-box domain representing Skp1-binding motif, normally in their amino-terminal portion, and contain a substrates-interacting domain in their carboxy-terminal region. They play the crucial role in recognising in a timely and specific manner a variety of substrates as an adaptor subunit of SCF (Skp1Cdc53/Cullin 1-F-box protein) ubiquitin ligases [8]. Beside this well-recognised function, an increasing number of F-box proteins has been described in partnership with Skp1 as non-SCF complexes [9]. For example, the budding yeast Rav2 F-box protein forms a complex with Skp1 and Rav1 and plays a role in vacuolar ATPase. It was recently shown that this complex appears to be conserved at least in fission yeast [10].

The $S$. pombe genome contains 18 genes encoding F-box proteins [9, 11]. Among the fission yeast F-box proteins, only two are essential, Pofl and Pof6 [12, 13]. While Pof 1 follows the traditional pattern as a component of $\mathrm{SCF}^{\text {Pof1 }}$, a counterpart of the budding yeast $\mathrm{SCF}^{\mathrm{Met30}}$ ubiquitin ligase [13], Pof6 seems to be less canonical. Pof6 was first identified as a Skp1 interactor and no binding to cullins was reported, bringing up the idea of a non-SCF complex [12]. In this study we isolated by TAP purification and MudPIT analysis a novel interactor of Pof6, named Sip1 (see below). We report characterisation of this novel type of the non-SCF complex that plays an essential role in endocytosis and cytokinesis.

\section{EXPERIMENTAL}

\section{Yeast strains and construction of sip1 temperature sensitive alleles}

Yeast strains used in this study are described in Table I. Cells were grown in standard culture media, and standard yeast genetic methods were used. DNA fragments containing error-prone PCR amplified $\operatorname{sipl}^{+}-H A$ sequences with the G418 resistance 
$\mathrm{kan}^{r}$ gene were transformed into a wild type strain and 6 temperature sensitive (ts) alleles were isolated (see Supplementary methods for details).

\section{TAP purifications}

The TAP purifications were mainly prepared as described previously [14]. For Pof6TAP purification, $40 \mathrm{~L}$ of cells were grown in YE5S at $30^{\circ} \mathrm{C}$ and disrupted in liquid nitrogen. Proteins were solubilised in the lysis buffer $(50 \mathrm{mM}$ Tris-HCl $\mathrm{pH} 8.0,150$ $\mathrm{mM} \mathrm{NaCl}, 5 \mathrm{mM}$ EDTA, $10 \%$ glycerol, $0.2 \% \mathrm{NP}-40,1 \mathrm{mM} \mathrm{NaF}, 10 \mathrm{mM}$ PMSF, adjusted to $\mathrm{pH}$ 8.0) and loaded for a two-step TAP purification. The eluted proteins were precipitated with TCA and subjected to MudPIT analysis (Multidimensional Protein Identification Technology, see Supplementary methods for experimental details). For Sip1-TAP purification, a total of $10 \mathrm{~L}$ of cells were cultured in YE5S at $30^{\circ} \mathrm{C}$ and disrupted in liquid nitrogen. The proteins were eluted in a single-step TAP purification and precipitated in TCA for further MudPIT analysis.

\section{Coimmunoprecipitation experiments}

Protein extracts were prepared in lysis buffer by breaking cells at $4{ }^{\circ} \mathrm{C}$ with glass beads (4 times for $40 \mathrm{~s}$ ) in a FastPrep FP120 apparatus (Savant, Co, MN, USA). The protein extracts were collected after $15 \mathrm{~min}$ of centrifugation at $10,000 \mathrm{x} \mathrm{g}$ at $4^{\circ} \mathrm{C}$. The coimmunoprecipitations were performed as described previously [15].

\section{Synchronisation by centrifugal elutriation and FACS analysis}

$3 \mathrm{~L}$ of YE5S media were inoculated with a preculture overnight. Elutriation was performed with Avanti J-20 XP (Beckman Coulter, MN, USA) by the standard procedures. Concentrations and sizes of cells were measured with a Cell Counter (Sysmex KX-21N, Kobe, Japan). Septa were stained with calcofluor and the percentage of septated cells were calculated by counting over a total of 100 cells. The cell survivals were estimated by spreading a calculated number of cells on YE5S plates and incubating them at $30^{\circ} \mathrm{C}$ for 3-4 days. Standard methods for FACS analysis were followed using a FACScan machine (Becton Dickinson, CA, USA).

\section{Fluorescence Microscopy}

For all microscopy experiments, cells were grown and observed in minimum medium at $27^{\circ} \mathrm{C}$, unless otherwise stated. Signals of GFP-Sip1 (expressed under the control of the thiamine-repressible $n m t l$ promoter) are essentially the same between in the presence (repressed) and absence (derepressed) of thiamine, though those in the absence of thiamine are stronger. For time-lapse analysis, therefore, derepressed conditions (-thiamine) were used as stronger signals were required for recording. Live cell imaging was performed in an imaging chamber (CoverWell, $20 \mathrm{~mm}$ diameter, 0.5 $\mathrm{mm}$ deep) (Molecular Probes) filled with $800 \mu 1$ of $2 \%$ agarose in minimum medium and sealed with a $22 \times 22 \mathrm{~mm}$ glass coverslip. FM4-64 (Molecular Probes Inc.) was dissolved in DMSO at a concentration of $1.64 \mathrm{mM}$ and added to the cells at a final concentration of $1.89 \mu \mathrm{M}$. Cells were then incubated in the dark before being applied to the imaging chamber. Cells were imaged using an Olympus IX71 microscope with a $63 X$ oil immersion lens, NA=1.4. Images were captured with a Coolsnap HQ digital CCD camera (Roper Scientific). Counts and measurements were made using Metamorph (Molecular Devices Corporation) and downloaded to Microsoft Excel for analysis. 


\section{RESULTS}

\section{Identification of a novel interactor of the Pof6 F-box protein}

To identify Pof6 interactors, we have performed a non-stringent Pof6-TAP purification in two steps from a large-scale culture $(40 \mathrm{~L})$ and coupled it to MudPIT analysis. A protein migrating at the size of Pof6 is specifically detected in the silver stain of Pof6-TAP elution (Figure 1A). Indeed the MudPIT data reveals a high coverage of Pof6 (19 unique peptides covered 56.2\% the sequence) considering the size of the protein, $99.9 \mathrm{kDa}$ (Supple. Figure S1A). As predicted, Skp1 appears in the elution with $58.4 \%$ coverage and a total of 30 peptides. The specificity of the interaction between Pof6 and Skp1 was confirmed by coimmunoprecipitations with both proteins (Figure 1B) as reported previously [12].

An uncharacterised ORF (SPBC27B12.08) was identified as one of the most abundant proteins pulled down in Pof6-TAP purification. Although the corresponding protein has the large size of $217 \mathrm{kDa}$, the peptide coverage reaches up to $60.6 \%$ of the sequence and a total of 792 peptides were isolated (Supple. Figure S1A). The specificity of the interaction between Pof6 and this novel protein was confirmed by coimmunoprecipitation (Figure 1C). We, consequently, named the protein according to that specific feature, Sip1 (pof $\underline{S} i x$ interactor protein 1).

In a reciprocal experiment, we performed a single step Sip1-TAP purification from a $10 \mathrm{~L}$ culture to identify its binding partners (Figure 1D). One of the most abundant proteins identified by MudPIT analysis is Skp1 (Supple. Figure S1B). The peptides cover $66.5 \%$ of Skp 1 sequence and a total of 35 peptides are present. The Fbox protein Pof6 is also detected as an interactor of Sip 1 albeit with a much restricted coverage $(6.1 \%)$. The difference between Pof6 coverage in Sip1-TAP elution and Sip1 coverage in Pof6-TAP elution may be explained by the amount of cells used for the purifications, the differences in the TAP purification protocols and the abundance/instability of each protein. To confirm the interactions observed by Sip1TAP MudPIT analysis, Sip1-HA was immunoprecipitated. As shown in Figure 1E, Pof6 and Skp1 were specifically pulled down with Sip1-HA.

We then attempted to examine whether Pof6 and Sip1 could interact with each other in the absence of Skp1. For this purpose, an Skp1 shut-off strain was used. In this strain, the thiamine-repressible nmtl promoter was inserted in front of the initiation codon of the genomic $s k p 1^{+}$gene together with HA epitope, by which the expression of HA-Skp1 could be shut off by adding thiamine to the medium [16]. Rather unexpectedly we found that when Skp1 levels were downregulated, both Sip1 and Pof6 became unstable, and accordingly we could barely detect either protein by immunoblotting (Figure 1F). Thus despite being not conclusive, this result indicates that Pof6 and Sip1 requires Skp1 for their stability, supporting the notion that these three proteins form a ternary complex in the cell.

It is of note that none of the two MudPIT datasets contains peptides for cullins (Supple. Figure S1) confirming the absence of interaction between Pof6 and the cullins as previously reported [12]. We made a careful visual inspection of the sequence corresponding to the Pof6 F-box domain and aligned it with different F-box domains present in SCF complexes [17, 18] (Supple. Figure 2A). The alignment shows that several residues important for the Skp1/F-box domain interface are present in Pof6 but the two residues involved in the Cul1/F-box domain core interface are not conserved; a highly conserved proline residue is replaced by threonine in Pof6 and an acidic residue is exchanged with asparagine. These residues have been shown to be specifically responsible for the interaction between Skp2 F-box protein and Cul1 [18], 
substantiating our and previous results [12] showing that Pof6 does not interact with cullins.

The analysis of the Pof6 protein also reveals the presence of unusual domains in its carboxy-terminal region: two Sec10 domains and a CAAX sequence are present in the substrate-binding portion (Supple. Figure 2B). Such features suggest that there may be potential functional similarities between Pof6 and Rcyl from budding yeast. Like Pof6, Rcy1 interacts with Skp1 and contains two Sec10 domains and a CAAX sequence in its carboxy-terminal region. In addition, it has been suggested that Rcyl is part of a non-SCF complex [19]. These results collectively suggest that Pof 6 does not belong to a conventional SCF complex but instead forms a novel complex with Sip1 and Skp1.

\section{The sip $1^{+}$gene is essential in $S$. pombe and conserved amongst eukaryotes}

To investigate Sip1 function, we asked whether the sip $1^{+}$gene is essential in $S$. pombe. We deleted by PCR one copy of the genes with a G418-resistant cassette in a

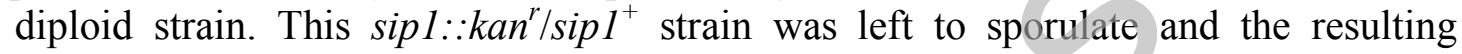
tetrads were dissected to examine the viability of each haploid progeny. Only two out of four haploids grew into colonies after germination, and these viable colonies were sensitive to G418 (Figure 2A, left panel). We concluded that sip $1^{+}$, like $p o f 6^{+}$and $s k p 1^{+}$, is essential for cell viability. A close observation of the non-viable spores showed that germinations occur but not cell division (Figure 2A, right panel).

Homology search reveals that Sip1 contains several HEAT repeats (closed boxes in Figure 2B), which are implicated in protein-protein interactions and often found in subunits of multi-protein complexes [20]. These HEAT repeats are distributed widely along the whole Sip1 protein (Figure 2B). Interestingly, Sip1 protein appears to be conserved throughout the evolution from yeasts to human beings (Figure 2C). Homologies are observed in not only HEAT repeats but also other regions as well.

\section{Sip1 plays an essential role in cytokinesis}

To examine the function of Sip1, we produced ts alleles of sip $1^{+}$by mutagenic PCR (sip1-62, see Supplementary methods for experimental details). Centrifugal elutriation was performed to synchronise cell cultures in early G2. Wild type and the sip1-62 ts cells were grown at $25^{\circ} \mathrm{C}$, elutriated and then shifted to $36^{\circ} \mathrm{C}$. sip1-62 ts cells ceased division almost immediately and their viability decreased concomitantly with the increase of septated cells upon mitotic exit (40-60 min, Figure 3A). In addition unlike wild type cells, the percentage of septated cells did not decline afterwards once it reached a plateau. Consistent with this, the population of the binucleate cells closely followed the profile of the septated cells. These observations suggest that sipl ts cells proceed through the G2 phase and enter mitosis to lose viability during cytokinesis. The DNA content of the synchronised cells showed that sip1-62 ts cells arrest with a $4 C$ DNA content, indicating a completion of DNA replication in each separated nucleus (Figure 3B).

We further investigated the role of Sip1 in cell division by following the fate of the CAR and septum in sip 1-62. The CAR was visualised using the type II myosin heavy chain protein, Myo2, tagged with GFP [6, 21]. Cells were grown at the permissive temperature, shifted up to the restrictive temperature for $1 \mathrm{hr}$, stained with calcofluor and observed at $36^{\circ} \mathrm{C}$ over-time (Figure 4 and Supp Movies S1 and S2). In the sipl mutant, the initiation timing of CAR and septum formation appeared very similar to that of wild type cells (Figure 4A and B). However, the CAR took nearly 
twice as much time to constrict. Although in the sip1 mutant, much variability was observed from one cell to the other in the timing of Myo2-GFP detachment, it was delayed ascribable to a prolonged CAR constriction process at the medial region (Figure 4B). In addition, septum materials remained at the equator. We could not even observe the execution of this process within the timeframe of experiments. Therefore Sip1, a newly identified conserved protein, plays an essential role in cytokinesis, in which it is required for CAR constriction and septum dissolution.

\section{Sip1 localises to the nucleus and endocytic vesicles}

To investigate the localisation of Sip1, we constructed a strain in which $\operatorname{Sip} 1$ was chromosomally tagged with GFP at its N-terminus and expressed under the nmt 1 promoter. GFP-Sip1 fluorescence was detected in the nucleus as well as in cytoplasmic punctuate structures of various sizes and shapes (Figure 5). Time-lapse imaging showed that these dots were capable of fusing and fragmenting and were also highly motile (Figure 5A and Supple. Movie S3). Tracking of individual patches over time revealed two types of motile behaviour: a local, possibly, Brownian movement, and a long-range linear displacement (Figure 5A and B). Both movements were observed independently of the stage of the cell cycle (not shown). It is clear however that the GFP-Sip1 dots used the long-range movement to accumulate during nuclear separation at the position of the future division plane (Figure 5A-C). Threedimensional reconstructions of wide-field microscopy images showed that the GFPSip1 fluorescence lined up on both sides of the primary, calcofluor-stained septum. They did not comprise rings or plates, but instead remained as the form of dots (Supple. Movie S4).

To evaluate the possibility that the Sip 1 patches represent Golgi vesicles, we constructed a strain containing GFP-Sip 1 and mCherry-tagged Anp1 or Sec72, a cisGolgi or trans-Golgi marker protein, respectively (kindly provided by A. Vjestica and S. Olifelenko) [22]. GFP-Sip1 did not colocalise with Anp1-mCherry, and only very partially did so with Sec72-mCherry (Supple. Figure S3), indicating that Sip1 dots do not represent Golgi organelles. We next set out to examine whether GFP-Sip1 patches were stained with the amphiphilic dye FM4-64 [23, 24]. In fission yeast, a 5-30 min incubation of the cells with this dye allows the visualization of endocytic vesicles that are being transported from the plasma membrane to the vacuolar membrane [25]. Under these conditions, we found that FM4-64 clearly stained GFP-Sip1-containing structures (Figure 6A). To test if Sip1 has any role in endocytosis, we incubated wild type and sip1-62 cells with FM4-64 for up to 90 min. Contrary to the situation in wild type cells where the dye was delivered to vacuoles by endocytosis, in the sip1-62 mutant, FM4-64 remained localising to the tips in growing cells, or to the equator in dividing cells, that is the sites where endocytosis should have occurred (Figure 6B). It is worth pointing out that the endocytosis defect was observed even at the permissive temperature, but was most strikingly obvious at the restrictive temperature. Taking all together, we conclude that Sip1 is a novel component of endocytic vesicles and essential for endocytosis and the completion of cytokinesis.

\section{DISCUSSION}

In this report, we identify a novel protein, Sip1, as a component of the Pof6/Skp1 complex in $S$. pombe. Immunoprecipitation experiments conform physical interactions between Sip1 and Pof6-Skp1. These three proteins appear to form a functional ternary complex, as in addition to coimmunoprecipitation data, we have found that protein 
stability of Sip1 and Pof6 is greatly compromised in the absence of Skp1, the phenomenon often observed in multi-subunit complexes. Given that a canonical SCF is a ubiquitin ligase in which stability of F-box proteins is often dependent upon structural integrity of a holo-SCF complex [9], this instability might imply that the Skp1-Pof6-Sip1 complex is involved in ubiquitin-mediated reactions. Sip1 is essential and conserved amongst eukaryotes. This protein consists of a number of HEAT repeats, protein-protein interaction motifs, implying that Sip1 plays a scaffolding role in this ternary complex. As none of cullins have been identified in TAP-purification (this study and [12]), it is likely that Skp1, Pof6 and Sip1 constitute an essential nonSCF F-box complex.

Creation and analysis of a sip1-62 ts strain have unravelled an essential role for Sip1 in cytokinesis and endocytosis. It is worth mentioning that a ts skp 1-3 allele and pof6-deleted haploid cells germinating from heterozygous diploids exhibited cytokinetic defects [12], reminiscent of the terminal phenotype of sip1-62 cells reported in this study. Sip1 localises to the nucleus and endocytic vesicles. Consistent with an interaction, Pof6 localises to the nucleus [12]. However, their cellular localisation is not completely parallel. In addition to the nucleus, Pof6 localises to cell tips during interphase and the CAR upon cytokinesis $[12,26]$, not to endocytic vesicles. It should, however, be noted that GFP-Sip1 vesicles appear to constantly gather around the CAR during CAR formation, which might reflect a physical interaction between these two proteins.

One of the most important results presented in this study is the identification of Sip1 as an essential factor for endocytosis. Cytokinesis is a complex process, in which a number of intra- and extra-cellular signalling pathways are involved and converged [27] including membrane trafficking [28]. Despite its importance, compared to our understanding of CAR formation and function, knowledge with regards to roles and mechanics of endocytosis in cytokinesis is still limited. Our study uncovers a novel component for endocytic vesicles, which will provide a tool by which to pursue the molecular pathways underlying endocytosis-mediated cytokinesis and cell separation.

Analysis of orthologues for Pof6 and Sip1 has been reported only in budding yeast. Like Pof6, F-box protein Rcy1 binds Skp1 and interestingly forms a non-SCF complex. Rcy1 participates in protein recycling from the endosomes to the Golgi and the plasma membrane $[19,29]$. However, unlike Pof6, Rcy1 is non-essential and these two proteins may not perform identical molecular functions $[9,12]$. So far we are not able to detect Pof6 orthologues by homology search in higher eukaryotes. The identification of functional counterparts of Pof6 in higher eukaryotes would be crucial to delineate their conserved roles. In the case of Sip1, its budding yeast orthologue is called Laa1. Like Rcy1 and unlike Sip1, Laa1 is non-essential [30]. Although no physical interaction between Rcyl and Laal is known, similar to Rcy1, Laal is reportedly involved in clathrin-mediated transport between trans-Golgi network and endosomes, which displays some analogy to Sip1 functions.

In conclusion, we describe the first non-SCF complex in $S$. pombe. We show that the Skp1-Pof6-Sip1 complex plays an essential role in cell division. Further analyses point towards a precise role for this complex in endocytosis and cytokinesis. It would be now vital to explore a role of Sip1 in membrane trafficking and identify functional pathways in which the Sip1-Pof6-Skp1 complex is involved. Investigation of roles for Sip1 orthologues in higher eukaryotes would be the next phase of our research. Finally, besides Pof6, there are a number of F-box proteins in both fission yeast and other organisms, in which two critical proline and acidic residues are not 
conserved [7]. We envision that non-SCF F-box complexes are more general than currently recognised and the elucidation of the biological functions of these novel complexes is a key challenge in the future.

\section{ACKNOWLEDGEMENTS}

We thank Dan Mulvihill for a Myo2-GFP strain, Aleksandar Vjestica and Snezhana Oliferenko for Golgi marker strains and Kathy Gould for TAP-tagging plasmids. We are grateful to Damien Hermand for exchanging unpublished information. We thank the Fermentation Unit of Cancer Research UK for preparations of large-scale fission yeast cultures. The work was supported by Cancer Research UK (T. T.) and National Institutes of Health grant P41RR11823. (J.R.Y.) 


\section{REFERENCES}

1 Barr, F. A. and Gruneberg, U. (2007) Cytokinesis: placing and making the final cut. Cell. 131, 847-860

2 Rajagopalan, S., Wachtler, V. and Balasubramanian, M. (2003) Cytokinesis in fission yeast: a story of rings, rafts and walls. Trends Genet. 19, 403-408

3 Guertin, D. A., Trautmann, S. and McCollum, D. (2002) Cytokinesis in eukaryotes. Microbiol. Mol. Biol. Rev. 66, 155-178

4 Le Goff, X., Utzig, S. and Simanis, V. (1999) Controlling septation in fission yeast: finding the middle, and timing it right. Curr. Genet. 35, 571-584

5 Feierbach, B. and Chang, F. (2001) Cytokinesis and the contractile ring in fission yeast. Curr. Opin. Microbiol. 4, 713-719

6 Wu, J. Q., Kuhn, J. R., Kovar, D. R. and Pollard, T. D. (2003) Spatial and temporal pathway for assembly and constriction of the contractile ring in fission yeast cytokinesis. Dev. Cell. 5, 723-734

7 Bai, C., Sen, P., Hofmann, K., Ma, L., Goebl, M., Harper, J. W. and Elledge, S. J. (1996) SKP1 connects cell cycle regulators to the ubiquitin proteolysis machinery through a novel motif, the F-box. Cell. 86, 263-274

8 Patton, E. E., Willems, A. R. and Tyers, M. (1998) Combinatorial control in ubiquitin-dependent proteolysis: don't Skp the F-box hypothesis. Trends Genet. 14, 236-243

9 Hermand, D. (2006) F-box proteins: more than baits for the SCF? Cell Div. 1, 30

10 Dawson, K., Toone, W. M., Jones, N. and Wilkinson, C. R. (2008) Loss of regulators of vacuolar ATPase function and ceramide synthesis results in multidrug sensitivity in Schizosaccharomyces pombe. Eukaryot. Cell. 7, 926-937

11 Lehmann, A., Katayama, S., Harrison, C., Dhut, S., Kitamura, K., McDonald, N. and Toda, T. (2004) Molecular interactions of fission yeast Skp1 and its role in the DNA damage checkpoint. Genes Cells. 9, 367-382

12 Hermand, D., Bamps, S., Tafforeau, L., Vandenhaute, J. and Makela, T. P. (2003) Skp1 and the F-box protein Pof6 are essential for cell separation in fission yeast. J Biol Chem. 278, 9671-9677

13 Harrison, C., Katayama, S., Dhut, S., Chen, D., Jones, N., Bahler, J. and Toda, T. (2005) SCF ${ }^{\text {Pofl }}$-ubiquitin and its target Zip1 transcription factor mediate cadmium response in fission yeast. EMBO J. 24, 599-610

14 de Bruin, R. A., McDonald, W. H., Kalashnikova, T. I., Yates, J., 3rd and Wittenberg, C. (2004) Cln3 activates G1-specific transcription via phosphorylation of the SBF bound repressor Whi5. Cell. 117, 887-898

15 Spielewoy, N., Flick, K., Kalashnikova, T. I., Walker, J. R. and Wittenberg, C. (2004) Regulation and recognition of SCF $^{\text {Grrl }}$ targets in the glucose and amino acid signaling pathways. Mol Cell Biol. 24, 8994-9005

16 Yamano, H., Kitamura, K., Kominami, K., Lehmann, A., Katayama, S., Hunt, T. and Toda, T. (2000) The spike of S phase cyclin Cig2 expression at the G1-S border in fission yeast requires both APC and SCF ubiquitin ligases. Mol Cell. 6, $1377-1387$

17 Schulman, B. A., Carrano, A. C., Jeffrey, P. D., Bowen, Z., Kinnucan, E. R., Finnin, M. S., Elledge, S. J., Harper, J. W., Pagano, M. and Pavletich, N. P. (2000) Insights into SCF ubiquitin ligases from the structure of the Skp1-Skp2 complex. Nature. 408, 381-386 
18 Zheng, N., Schulman, B. A., Song, L., Miller, J. J., Jeffrey, P. D., Wang, P., Chu, C., Koepp, D. M., Elledge, S. J., Pagano, M., Conaway, R. C., Conaway, J. W., Harper, J. W. and Pavletich, N. P. (2002) Structure of the Cul1-Rbx1-Skp1-F boxSkp2 SCF ubiquitin ligase complex. Nature. 416, 703-709

19 Galan, J. M., Wiederkehr, A., Seol, J. H., Haguenauer-Tsapis, R., Deshaies, R. J., Riezman, H. and Peter, M. (2001) Skplp and the F-box protein Rcylp form a nonSCF complex involved in recycling of the SNARE Snclp in yeast. Mol. Cell. Biol. 21, 3105-3117

20 Neuwald, A. F. and Hirano, T. (2000) HEAT repeats associated with condensins, cohesins, and other complexes involved in chromosome-related functions. Genome Res. 10, 1445-1452

21 Mulvihill, D. P. and Hyams, J. S. (2003) Role of the two type II myosins, Myo2 and Myp2, in cytokinetic actomyosin ring formation and function in fission yeast. Cell Motil. Cytoskeleton. 54, 208-216

22 Vjestica, A., Tang, X. Z. and Oliferenko, S. (2008) The actomyosin ring recruits early secretory compartments to the division site in fission yeast. Mol. Biol. Cell. 19, 1125-1138

23 Vida, T. A. and Emr, S. D. (1995) A new vital stain for visualizing vacuolar membrane dynamics and endocytosis in yeast. J. Cell Biol. 128, 779-792

24 Bone, N., Millar, J. B., Toda, T. and Armstrong, J. (1998) Regulated vacuole fusion and fission in Schizosaccharomyces pombe: an osmotic response dependent on MAP kinases. Curr. Biol. 8, 135-144

25 Gachet, Y. and Hyams, J. S. (2005) Endocytosis in fission yeast is spatially associated with the actin cytoskeleton during polarised cell growth and cytokinesis. J. Cell Sci. 118, 4231-4242

26 Matsuyama, A., Arai, R., Yashiroda, Y., Shirai, A., Kamata, A., Sekido, S., Kobayashi, Y., Hashimoto, A., Hamamoto, M., Hiraoka, Y., Horinouchi, S. and Yoshida, M. (2006) ORFeome cloning and global analysis of protein localization in the fission yeast Schizosaccharomyces pombe. Nat. Biotechnol. 24, 841-847

27 Pollard, T. D. (2008) Progress towards understanding the mechanism of cytokinesis in fission yeast. Biochem. Soc. Trans. 36, 425-430

28 Albertson, R., Riggs, B. and Sullivan, W. (2005) Membrane traffic: a driving force in cytokinesis. Trends Cell Biol. 15, 92-101

29 Lafourcade, C., Galan, J, M., Gloor, Y., Haguenauer-Tsapis, R. and Peter, M. (2004) The GTPase-activating enzyme Gyplp is required for recycling of internalized membrane material by inactivation of the Rab/Ypt GTPase Ypt1p. Mol. Cell. Biol. 24, 3815-3826

30 Fernandez, G. E. and Payne, G. S. (2006) Laalp, a conserved AP-1 accessory protein important for AP-1 localization in yeast. Mol Biol Cell. 17, 3304-3317 


\section{FIGURE LEGENDS}

\section{Figure 1: Identification of a novel interactor of Pof6}

(A) TAP purifications of a non-tagged control strain (Table 1) and Pof6-TAP. Fractions corresponding to $1 / 100$ of each elution (E) were loaded in parallel to $100 \mu \mathrm{g}$ of total extract (TE) and silver-stained. Two bands migrating around $220 \mathrm{kDa}$ and 100 $\mathrm{kDa}$ (arrows) may correspond to Sip1 and Pof6 respectively. (B) Coimmunoprecipitation of Pof6-TAP and Skp1. Proteins were immunoprecipitated (IP) with anti-TAP, anti-Skp1 or no antibodies (Ab). $100 \mu \mathrm{g}$ of whole cell extract (WCE) were loaded and $2 \mathrm{mg}$ of proteins were used for each IP. Pof6-TAP was immunodetected with anti-Pof6 antibodies and Skp1 with anti-Skp1 antibodies. (C) Coimmunoprecipitation of Pof6/Pof6-TAP and Sip1-HA. Extracts were immunoprecipitated with IgG Sepharose beads or anti-HA antibodies. Immunoprecipitates were blotted with anti-HA and anti-Pof6 antibodies. The asterisk indicates a non-specific band of the IgG. (D) Single step TAP purification of Sip1TAP. $1 / 100$ of the final elution (E) was loaded on the gel in parallel to $100 \mu \mathrm{g}$ of total extract (TE) and silver-stained. A band around $75 \mathrm{kDa}$ probably represents mixtures of heat shock 70 chaperone family (the coverage percentages of Ssa2, Ssc1 and Ssa1 are $80.2 \%, 68.8 \%$ and $51.7 \%$ respectively). (E) Coimmunoprecipitation of Sip1-HA, Pof6 and Skp1. Sip1-HA was immunoprecipitated with anti-HA antibodies and immuno-complex was blotted with individual antibodies. (F) Instability of Sip1 and Pof6 in the absence of Skp1. Whole cell extracts were prepared from a strain containing $n m t 1$-HA-Skp1, Sip1-HA and Pof6-TAP in the absence (left, derepressed, + for Skp1) or presence (right, repressed, - for Skp1) of thiamine. Skp1 and Sip1 were immunodetected with anti-HA antibody, whilst Pof6-TAP was detected with antiPof6 antibody.

\section{Figure 2: $\operatorname{sip}^{+}$is an essential gene present amongst eukaryotes}

(A) Tetrad dissection of asci produced by a sipl::kan ${ }^{r} /$ sip $^{+}$diploid strain. Spores were grown for 3 days at $30^{\circ} \mathrm{C}$ on rich media (left). Phase contrast micrographs showing the terminal morphology of germinating sipl-deleted spores (right). Bar indicates $5 \mu \mathrm{m}$. (B) Schematics of Sip1 protein domains. Sip1 protein is 1920 amino acids long and contains HEAT repeats (black). (C) Dendogram of Sip1 orthologues identified by YOGY search. Proteins are labelled with the UniProtKB nomenclature. The ClustalW alignment default parameters of MacVector 9.0 were used to create the tree. The evolutionary distance is indicated on each branch. S.p: Schizosaccharomyces pombe; D.m: Drosophila melanogaster; H.s: Homo sapiens; C.e: Caenorhabditis elegans; S.c: Saccharomyces cerevisiae.

\section{Figure 3: Characterisation of the sip1-62 mutant}

(A) Centrifugal elitriation. Wild type (top) or sip1-62 cells (bottom) were synchronised by elutriation at $25^{\circ} \mathrm{C}$, shifted to $36^{\circ} \mathrm{C}$ (T0) and grown for $4 \mathrm{~h}$. Samples were taken every $20 \mathrm{~min}$ to measure cell viability, septation index and cell numbers. (B) DNA content analysis of wild type and sip1-62. Each strain was grown and treated as described in (A) and samples were taken every 20 min interval, followed by FACS analysis.

\section{Figure 4: Cytokinesis is not completed in the sip1-62 mutant}


(A) Time-lapse imaging of Myo2-GFP. sip1 ${ }^{+}$and sip1-62 cells containing Myo2-GFP (green) were stained with calcofluor (blue) in liquid cultures. These cells were shifted from $25^{\circ} \mathrm{C}$ to $36^{\circ} \mathrm{C}$ for $1 \mathrm{~h}$ and live imaging started. Pictures were acquired at $2 \mathrm{~min}$ intervals for up to $2 \mathrm{~h}$. Every second picture of the cell medial plane is shown. Asterisks show the time when the septum appeared. Bar, $3 \mu \mathrm{m}$. See also Supple. Movies S1 and S2. (B) Graphics showing the timing of cytokinetic events as they occur in sip1 ${ }^{+}$(blue) and sip1-62 (orange) cells. Cells were prepared as in (A). For each strain, results represent the mean of two independent experiments and error bars are SD of the average. A total of 39 cells for the wild type, and 31 cells for sip 1-62 were counted. The time of septum dissolution in sip1-62 could not be accurately determined under our experimental conditions and is shown as an open diamond. Sept., septum; CAR, Cytokinetic Actomyosin Ring.

\section{Figure 5: Localisation tracking analysis of GFP-Sip1}

(A) Time-lapse imaging of cells expressing GFP-Sip1. The GFP fluorescence is detected in the nucleus and in punctuated structures, which accumulate at the medial plate of the cell. The time is indicated in min:sec and matches the time used in Supple. Movie S3. Red arrowheads, a patch being rapidly displaced towards the division plane. Note that this patch resulted from the fusion of at least 3 patches (e.g: compare times 12:30 and 14:00) (B) Trajectories of individual GFP-Sip1 patches movement. Each color represents a patch and each pattern represents the path followed by the patch over time. Open symbols (circles) indicate patches starting points. The path of the dot shown in (A) with the red arrowhead is shown in red in (B). Only patches that could unambiguously be followed for at least $4 \mathrm{~min}$ are shown. (C) Kymographs of a GFP-Sip1 cell stained with calcofluor. GFP-Sip 1 gathers at the site of the division plane when the nuclei separate. As soon as the septum forms (blue arrowhead), patches line lateral to it. See also Supp Movie S4. N, Nucleus. Bar, $3 \mu \mathrm{m}$.

\section{Figure 6. Sip1 localises to endocytic vesicles and is required for endocytosis}

(A) Localisation of GFP-Sip1 to endocytic vesicles. nmt1-GFP-Sip1 cells were stained for $5 \mathrm{~min}$ with FM4-64 and observed immediately before the dye was transported to the vacuoles. The enlargement (right panel) shows that both signals perfectly overlap. (B) Endocytosis defects in the sipl mutant. Wild type (sip1+) and sip 1-62 cells were stained for an extended time with FM4-64. Whilst in wild type, the dye localises to peripheries of individual vacuoles, in the mutant, this dye is not incorporated inside the cell and instead accumulates at the tips (plain arrowhead) and septum (open arrowhead). Bars, $5 \mu \mathrm{m}$. Enlargement bar, $1 \mu \mathrm{m}$. 
Table I: Strain list

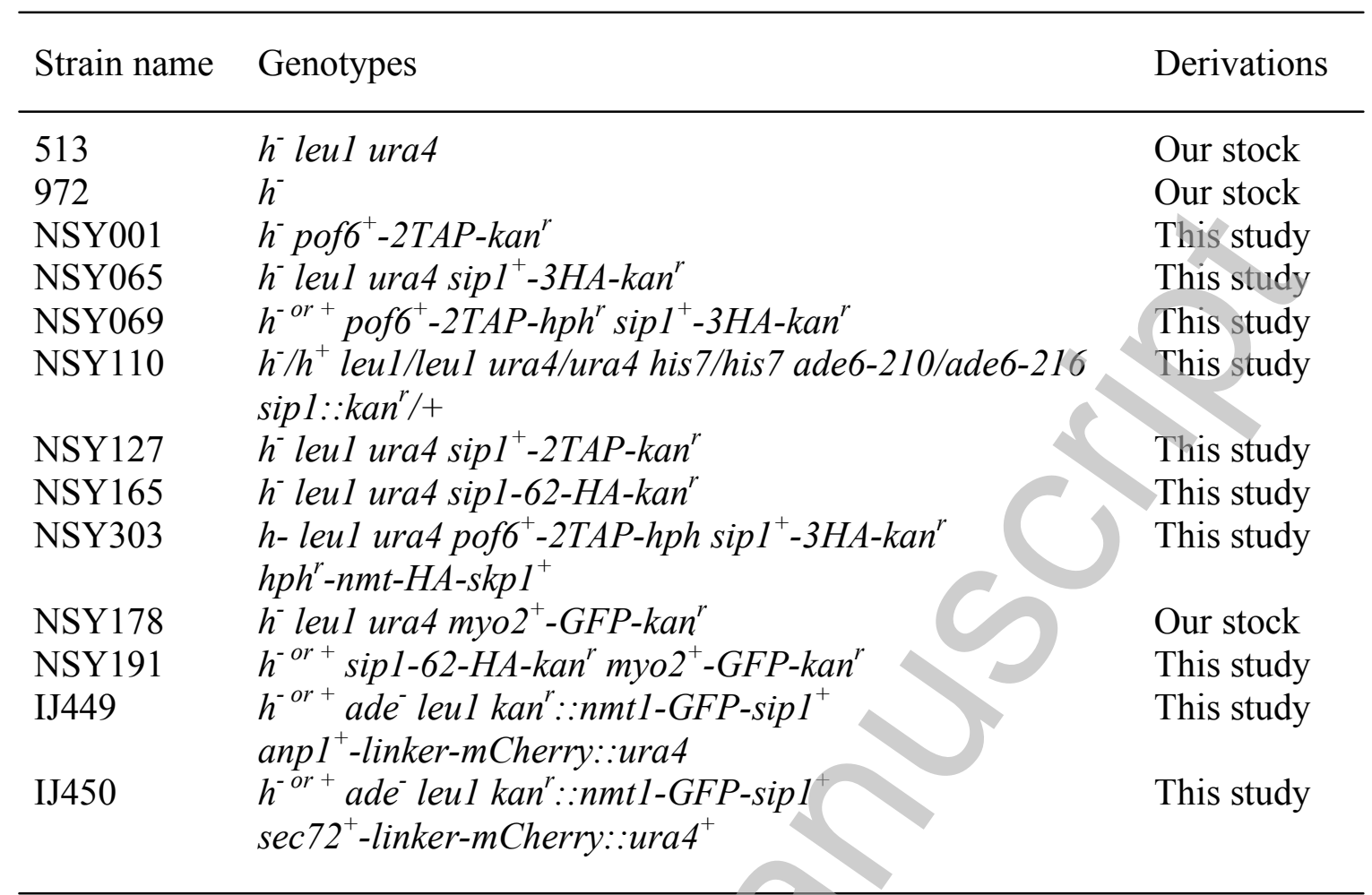




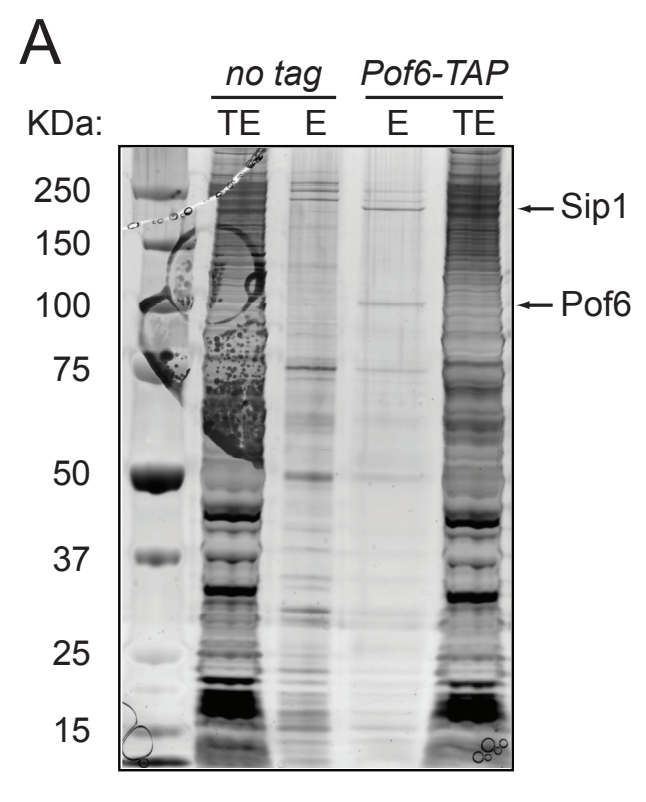

C

B

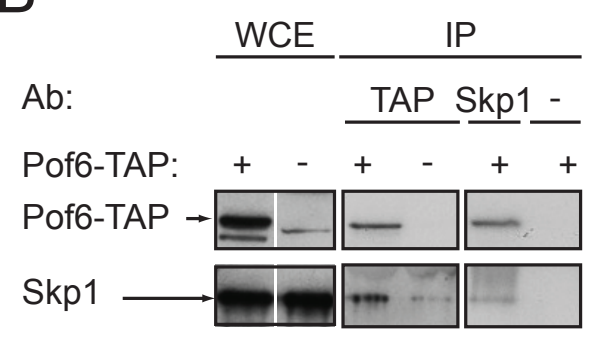

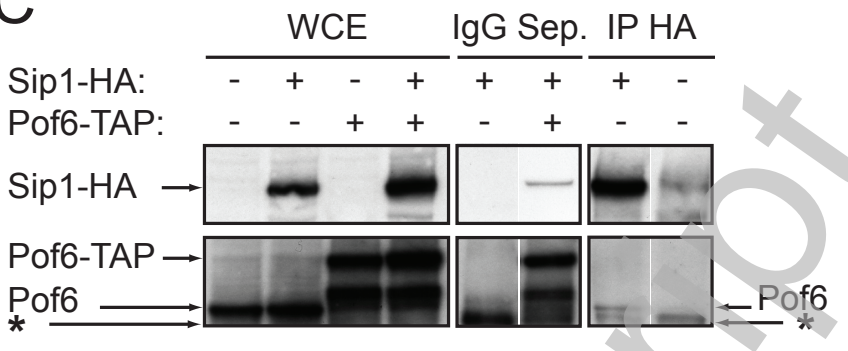

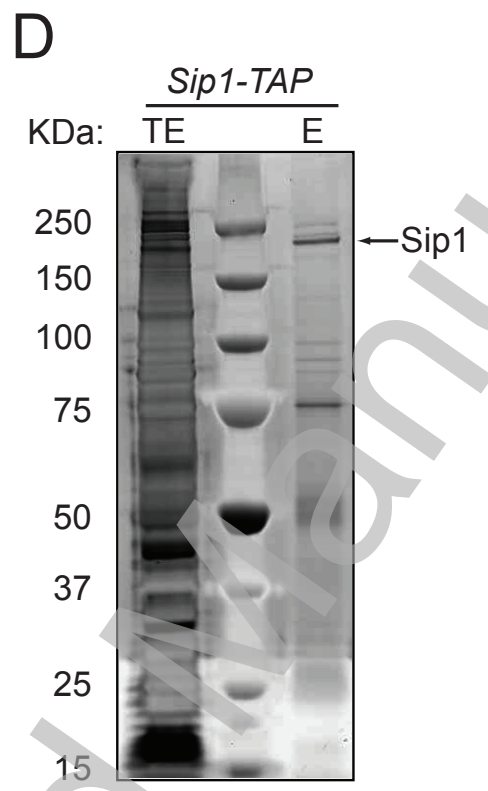

E
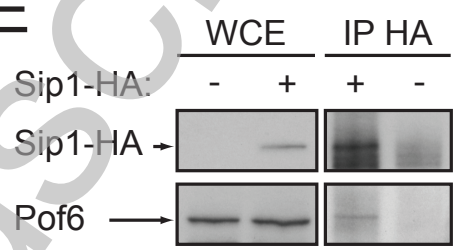

Skp1 $\leadsto-$

F

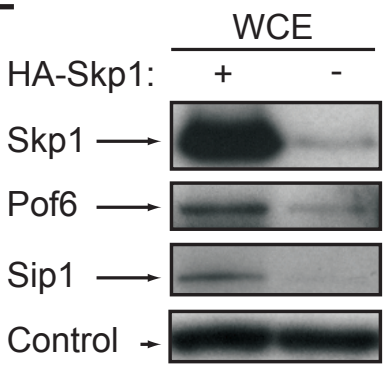

Figure 1: Jourdain et al. 
A

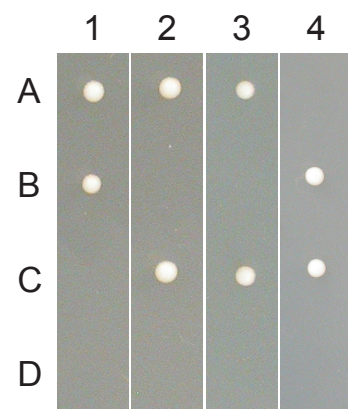

tetrad dissection
B

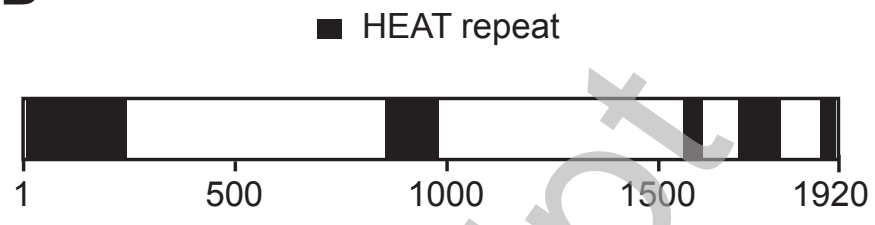

C

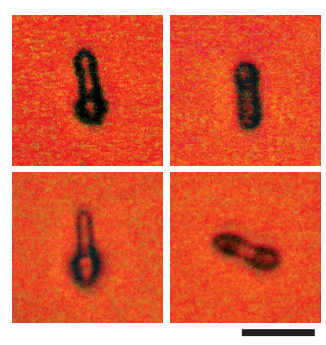

non-viable spores

Figure 2: Jourdain et al.

Licenced copy. Copying is not permitted, except with prior permission and as allowed by law. (C) 2009 The Authors Journal compilation @ 2009 Portland Press Limited 
A

- Cell survival

- Cell number

- Septation index

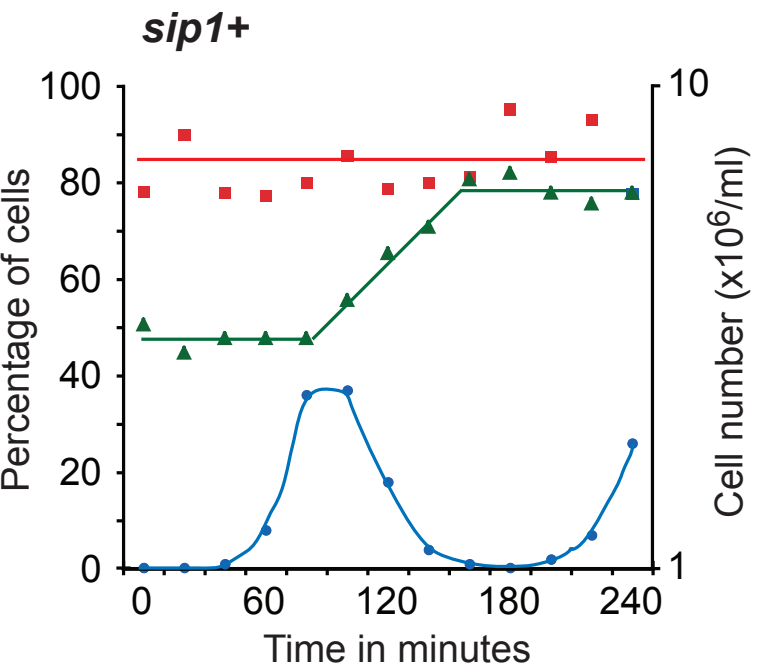

\section{sip1-62}

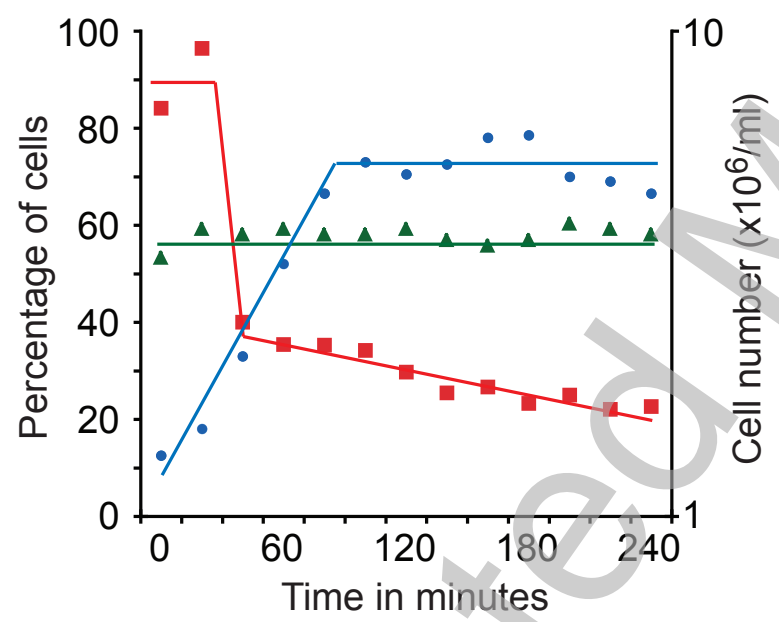

B

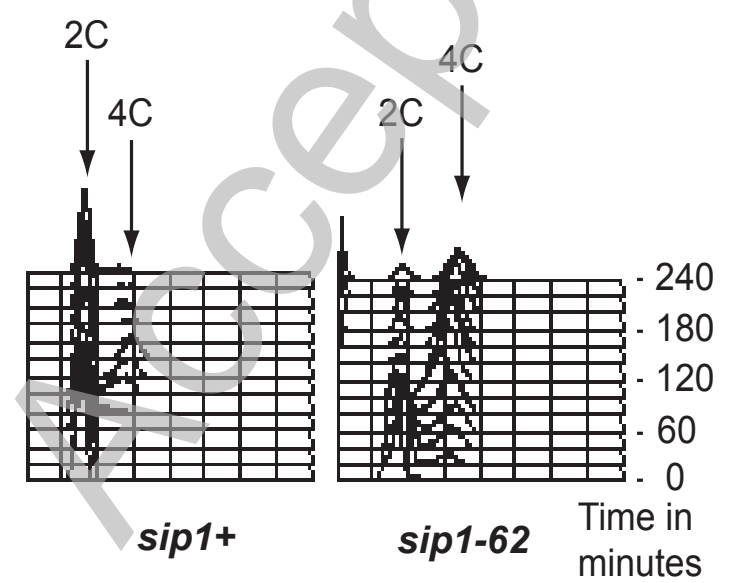

Figure 3: Jourdain et al. 
A

Time (min):

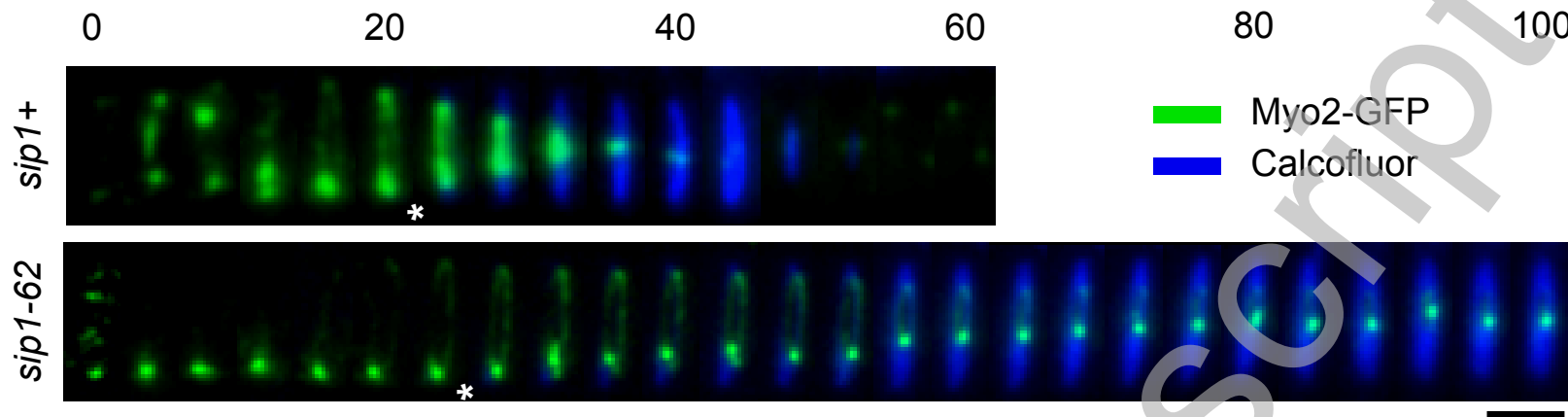

B

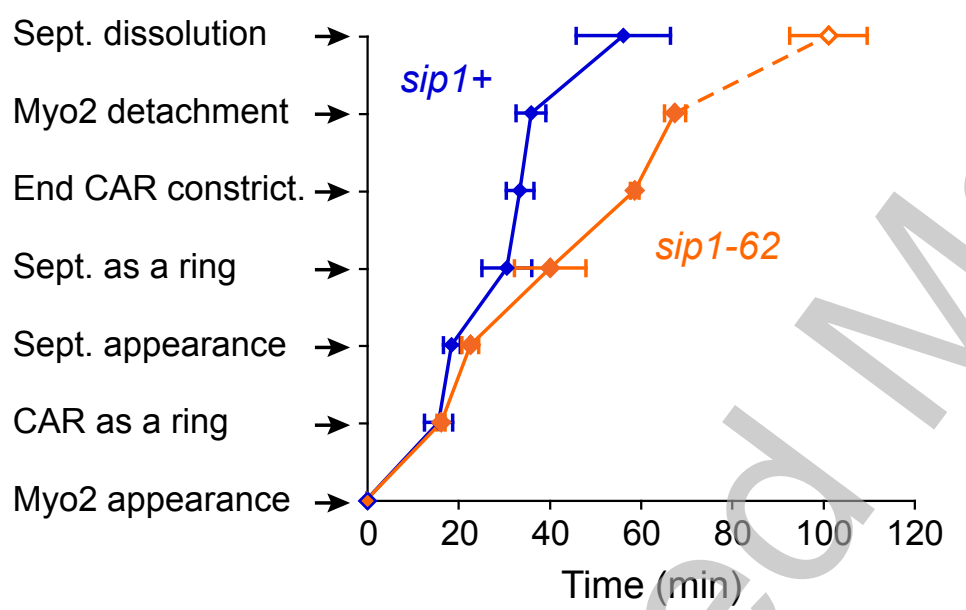

Figure 4: Jourdain et al.

Licenced copy. Copying is not permitted, except with prior permission and as allowed by law. (C) 2009 The Authors Journal compilation (c) 2009 Portland Press Limited 
A

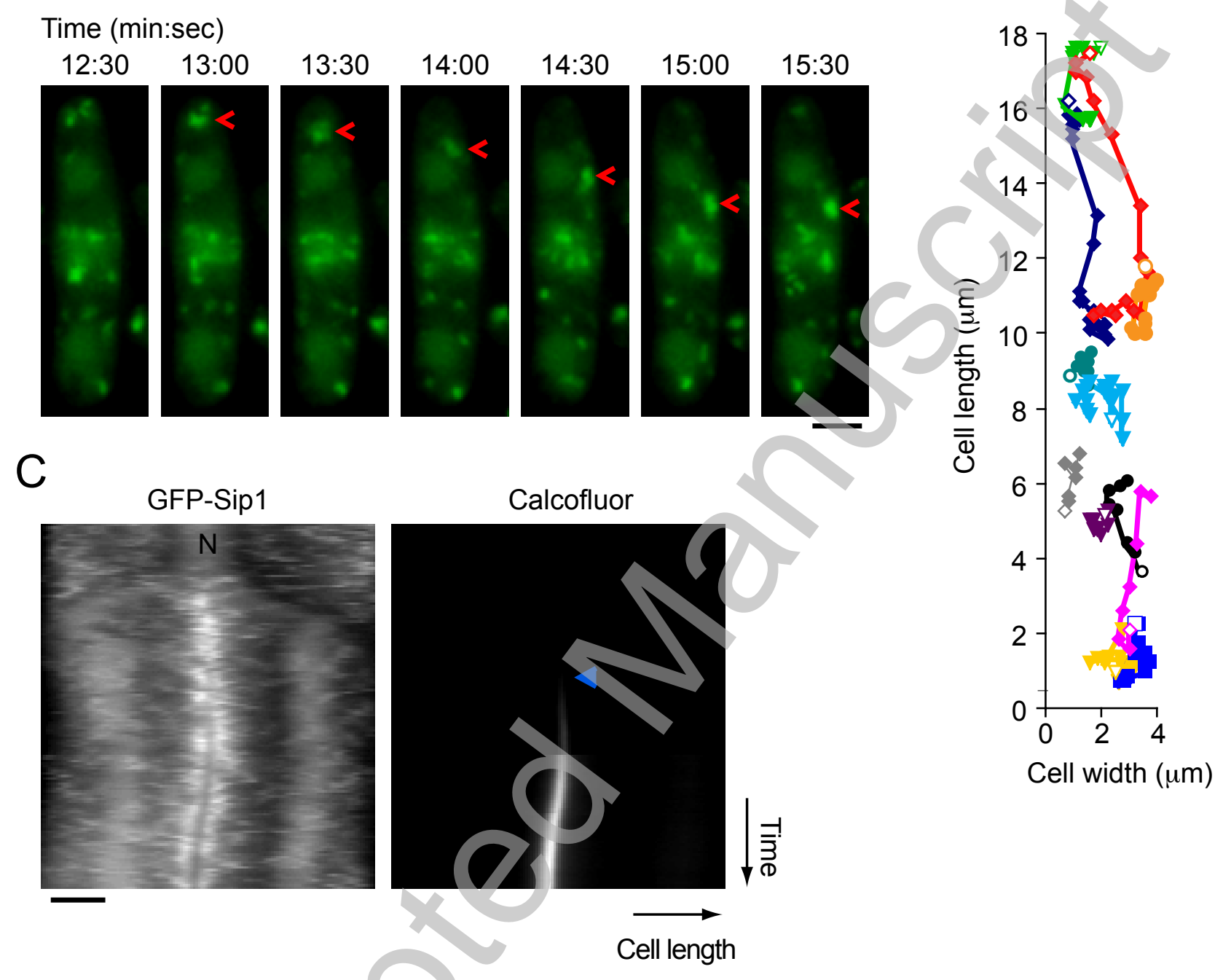

Figure 5: Jourdain et al. 
A
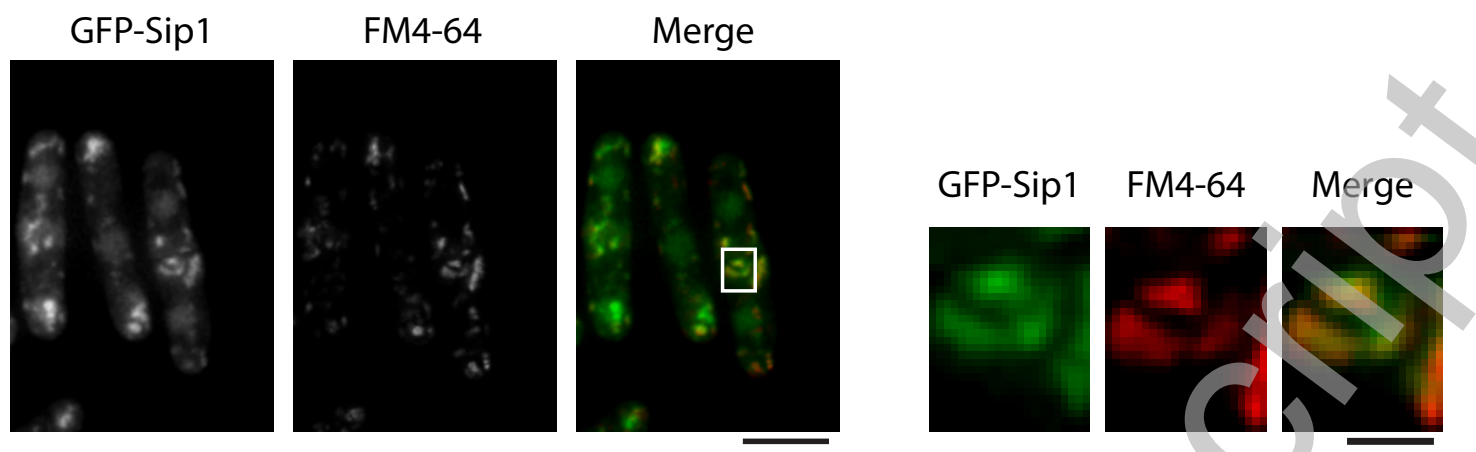

B

$\operatorname{sip} 1+$
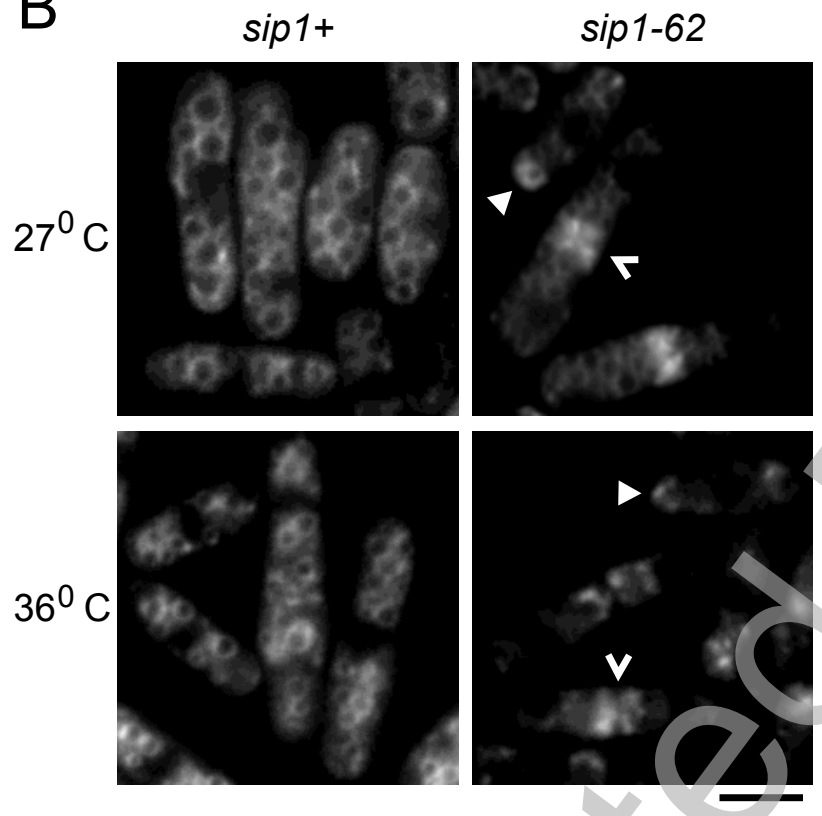

Figure 6: Jourdain et al.

Licenced copy. Copying is not permitted, except with prior permission and as allowed by law.

(C) 2009 The Authors Journal compilation (c) 2009 Portland Press Limited 